Bernard LAHIRE, Ceci n'est pas qu'un tableau. Essai sur l'art, la domination, la magie et le sacré

\title{
Marc Joly
}

\section{(2) OpenEdition}

\section{Journals}

Édition électronique

URL : http://journals.openedition.org/ress/3702

DOI : $10.4000 /$ ress.3702

ISBN : $1663-4446$

ISSN : 1663-4446

Éditeur

Librairie Droz

Édition imprimée

Date de publication : 15 mai 2017

Pagination : 272-275

ISSN : 0048-8046

Référence électronique

Marc Joly, «Bernard LAHIRE, Ceci n'est pas qu'un tableau. Essai sur l'art, la domination, la magie et le sacré ", Revue européenne des sciences sociales [En ligne], 55-1 | 2017, mis en ligne le 03 janvier 2017 , consulté le 19 janvier 2021. URL : http://journals.openedition.org/ress/3702 ; DOI : https://doi.org/ 10.4000/ress.3702

Ce document a été généré automatiquement le 19 janvier 2021.

(c) Librairie Droz 


\title{
Bernard LAHIRE, Ceci n'est pas qu'un tableau. Essai sur l'art, la domination, la magie et le sacré
}

\author{
Marc Joly
}

\section{RÉFÉRENCE}

Bernard LAHIRE, 2015, Ceci n'est pas qu'un tableau. Essai sur l'art, la domination, la magie et le sacré, Paris, La Découverte, «Laboratoire des sciences sociales », 598 p.

1 En 2007, le musée des Beaux-Arts de Lyon acquiert pour 17 millions d'euros un tableau attribué à Nicolas Poussin, La Fuite en Égypte. L'année suivante, la directrice du musée contacte Bernard Lahire, professeur de sociologie à l'ENS de Lyon. Elle l'incite à s'intéresser à l'histoire de ce tableau. Elle met à sa disposition un dossier de presse qu'on devine épais. De quoi est-il question?

D'une controverse, tout d'abord: il existe plusieurs versions (représentant la même scène biblique) d'un tableau peint par Poussin en 1657-1658. Celle achetée par le musée lyonnais - après que les propriétaires d'origine eurent recouvré leur bien qui avait été cédé comme une "copie d'époque ", lors d'une discrète vente aux enchères, à des galeristes parisiens décrits, avec empathie, comme les dindons de la farce - a finalement été reconnue par la plupart des experts (à la suite de Jacques Thuillier, professeur au Collège de France) comme " autographe ", c'est-à-dire comme la version originale, peinte de la main de l'artiste, au détriment d'une version détenue par une richissime collectionneuse nord-américaine, pourtant "publiée » au début des années 1980 par le plus réputé des spécialistes alors vivants du peintre français, l'énigmatique Anthony Blunt.

D'une success-story, ensuite : grâce à la mobilisation exemplaire du musée du Louvre et aux efforts conjugués de l'État, de la mairie de Lyon, de la région Rhône-Alpes et de nombreuses entreprises s'adonnant au mécénat, un «chef d'œuvre» du «plus grand 
peintre français de tous les temps » (p. 529) a pu demeurer sur le territoire national et venir orner les murs du musée d'une grande capitale régionale - et ce qui avait revêtu l'aspect d'une sorte de "guerre franco-britannique » (p. 391) a pu se clore sous le sceau de l'évidence, le prix d'achat et le consensus des experts se redoublant l'un l'autre pour produire un tel sentiment : « Quand on voit les tableaux l'un à côté de l'autre, s'exclame ainsi un responsable du musée lyonnais, on se demande même pourquoi il y a eu un doute un jour» (p. 417).

4 Ce feuilleton à rebondissement est présenté dans la troisième et dernière partie (« Du Poussin et de quelques Fuites en Égypte ») de Ceci n'est pas qu'un tableau. À la lecture, en particulier, du dernier chapitre («Comment chacun joue son jeu»), il ne fait apparemment aucun doute que la plupart des acteurs concernés de près ou de loin par l'acquisition publique de la toile se sont montrés parfaitement rationnels. Prenant à cœur leur métier, ils se sont appliqués à respecter la loi et à tenir compte des contraintes du marché. Ils se sont fixé des objectifs et ont fait en sorte de les atteindre. Ils possèdent une indéniable capacité d'analyse de leurs actions, non sans une conscience parfaitement ajustée de ce qui sied en matière de critique réflexive (les nombreux extraits d'entretiens cités en témoignent). C'est dire qu'il aurait été tentant, et commode, de prendre pour argent comptant leurs discours.

Or, insiste Lahire avec force, c'eût été ne pas apercevoir à quel point ils ont été, ils sont gouvernés, au fond, par un ensemble de croyances multiséculaires, dont deux lui paraissent déterminantes au plus haut degré : 1 . la croyance en l'art en tant qu'il se distingue du monde "profane " et relève du pôle du "sacré »; 2 . le culte porté au créateur individuel perçu comme l'égal de Dieu (et donc intégré parmi les puissants, et séparé des dominés, de tous ceux qui ne sont pas des créateurs) ou nanti d'une aura divine par le fait même de mettre au monde. De là, depuis le début du xix siècle, lorsqu'un doute affleure, cette obsession de l'attribution, du « raccordement » (d'une œuvre à un nom propre), ou de l'authentification, dont la nécessaire stabilisation dépend de l'autorité de ceux qui sont amenés à produire ce genre d'actes performatifs. Aux yeux du sociologue, une réflexion sur l'histoire objectivée, sur la " magie sociale ", sur les rapports de domination et sur la sociogenèse du sacré artistique était par conséquent inévitable. Elle se déploie dans deux parties très denses, la première revêtant une forme spinoziste ( Histoire, domination et magie sociale »), la seconde, une allure, disons, plus classique (« Art, domination, sacralisation »).

6 Pour l'auteur, la croyance dans le caractère sacré de l'art et la foi dans le génie créateur de l'artiste individuel forment, en quelque sorte, un "socle" sur lequel les comportements et les motivations des acteurs de l'achat du Poussin, et les controverses relatives à sa nature autographe, trouvent leur véritable support. Dès lors, leurs actions, leur vie, même, peuvent sembler un peu absurdes, voire dérisoires, baignées d'une magie (une "magie sociale ») grâce à laquelle, cependant, l'association à des valeurs éternelles et à plus grand que soi faisant son œuvre, la mort (leur mort) est apprivoisée, sublimée, pour ne pas dire magnifiée.

Ayant identifié un "socle de croyances ", Lahire le pense comme un "état de fait " (idéel mais aussi matériel) qui a une histoire, c'est-à-dire comme un état qui s'est fait, par sédimentation en quelque sorte. Cette histoire, selon lui, est celle de la structuration permanente des sociétés humaines en « dominants » et en "dominés », et de la contribution de l'art et des artistes à la mise en forme spécifique - datée et située, et rendue possible notamment par «la constitution de domaines artistiques distincts 
des domaines artisanaux» (p.529) - d'une opposition qui est issue de cette grande opposition structurante autant qu'elle la légitime : l'opposition sacré/profane. Donnant les clefs de cette histoire, en plusieurs étapes régressives, le sociologue est conduit à « désévidencier l'évident » (p. 61), et, fatalement, à « dérouter toutes les attentes de ceux qui, engagés à un degré ou à un autre dans un rapport pratique (institutionnel, politique, académique, économique, juridique, esthétique, etc.) à l'œuvre ne voient pas sur quel sol leurs pieds sont posés » (p. 365-366).

8 L'auteur, précisons-le, s'est surtout fait connaître comme sociologue travaillant à l'échelle des individus, comme théoricien de la pluralité des dispositions individuelles produites dans des contextes divers donnés et actualisées selon les cadres d'action fréquentés. Il n'a jamais cessé de plaider pour une variation des échelles d'observation en fonction des contraintes de l'objet et de la nature - déterminée à la fois par lesdites contraintes et par les centres d'intérêt et problématiques existentielles propres à tout chercheur - des problèmes théoriques à résoudre. Dans Ceci n'est pas qu'un tableau, il démontre qu'il n'a rien perdu de son intérêt pour les trajectoires individuelles. Le bref chapitre consacré au parcours et à la fortune de Poussin est dans l'esprit de son essai sur la création littéraire de Franz Kafka. D'autre part, l'essentiel est dit des expériences socialisatrices et des dispositions des principaux acteurs pris pour objet. Lahire a mené une enquête sérieuse. Seize entretiens ont été réalisés (dont dix par la sociologue Géraldine Bois). À propos des procès et controverses dont il fait état, il a pu réunir, en outre, des références de première main (lettres, mails, rapports divers, etc.) d'une richesse d'autant plus saisissante - certains documents appartenant indubitablement à la catégorie des « documents confidentiels " - qu'il s'agit d'une histoire récente et que la plupart des acteurs sont encore en vie. Cela dit, rendant hommage aux "grands fondateurs de nos disciplines » (Marx, Durkheim, Weber, Dumézil, Elias, Bourdieu) dans un post-scriptum intitulé "Les conditions de la création scientifique", Lahire revendique surtout le fait d'offrir au lecteur un travail de synthèse, une œuvre de seconde main nourrie de "travaux empiriques [surtout d'anthropologues et d'historiens] dont la qualité n'est pas sujette au doute » et qui lui auraient donné les moyens, à ce titre, de développer une vision plus ample que s'il était resté rivé « sur la monographie ou l'étude de cas "de première main" comme seule forme légitime d'administration de la preuve » (p. 556).

Ceci n'est pas qu'un tableau est un ouvrage imposant et extrêmement ambitieux. Il est aussi très déconcertant. Il est par exemple étrange, au vu, justement, du post-scriptum, que Lahire n'aborde pas expressément la question de la grandeur en science à la lumière du démontage effectué tout au long de son travail des opérations de construction sociale du «culte des grands maitres » (p. 259) en art. Pourquoi ce qui vaudrait comme règle générale pour les œuvres d'art ("sous l'admiration, la domination ») ne vaudrait-il pas pour les œuvres de science? «La création n'appelle pas la création, mais l'admiration du dominé pour le dominant » (p. 540), écrit l'auteur. Pourquoi la création, en science, ferait-elle exception? Que doit-on penser de l'admiration dont témoigne ouvertement Lahire pour ses "grands " prédécesseurs ? À notre avis, le problème aurait dû être traité au moins a minima - d'autant qu'il est assez facile à résoudre. On pourrait se demander simultanément - puisque l'auteur s'inscrit plus que jamais dans un sillage bourdieusien, jusque dans ses diatribes bien senties contre les propositions aporétiques de Luc Boltanski ou de Bruno Latour - si la théorie lahirienne centrale du "sacré » comme formule de soumission ne schématise pas quelque peu les notions bourdieusiennes d'arbitraire, de magie sociale et de violence 
sociale (par lesquelles, comme l'a bien montré Antoine Lentacker, l'auteur du Sens pratique s'est émancipé du structuralisme lévi-straussien). D'autre part, des historiens et sociologues de l'art ont relevé une volonté flagrante de non-confrontation avec les pairs (Du peintre à l'artiste de Nathalie Heinich n'est pas cité, par exemple). Sur un plan plus général, on regrettera que l'auteur laisse en suspens ou évite d'examiner des problèmes épistémologiques cruciaux, comme la dialectique modèle structural / modèle processuel ou la discrimination des invariants «naturels » et des invariants "sociaux ». Étant donné la nature de son propos, il aurait peut-être aussi gagné à se référer aux travaux de Maurice Godelier, qui, en particulier dans $L a$ Production des Grands Hommes, éclaire non seulement le problème des hiérarchies (en distinguant la domination générale des hommes sur les femmes des inégalités entre les hommes déterminées par l'héritage ou l'obtention par le «mérite» de certaines positions de pouvoir "ouvertes»), mais aussi la façon dont les représentations imaginaires contribuent à inscrire dans l'intimité corporelle l'ordre social dans ses multiples dimensions. Volontairement, sans doute, Lahire se tient à distance de la littérature anthropologique gouvernée par le paradigme du Big Man. Un « cheminement régressif» (p.366) sur le "mérite» n'aurait-il pas toutefois été des plus précieux (notamment parce qu'il aurait pu l'amener à croiser des œuvres importantes sur l'image du génie ou de l'artiste, comme celles d'Edgar Zilsel ou du duo Ernst Kris-Otto Kurz) ? Enfin, sur la forme, la manière dont Lahire discute les sociologues « classiques » (Weber, Durkheim, Bourdieu), et se situe par rapport à eux tout en s'en nourrissant avec un appétit d'ogre, n'est pas toujours satisfaisante. Quelquefois, il séduit par ses prolongements. D'autres fois, il laisse son lecteur franchement dubitatif (on pense, par exemple, à sa critique de la notion de «capital symbolique » [p. 81-85], qui reprend largement des précisions et des clarifications apportées par Bourdieu lui-même).

Mais ces critiques sont proportionnelles à l'ambition de l'auteur. On ne saurait trop conseiller à chacun de se faire sa propre opinion, et de partir à la rencontre d'un livre sans équivalent dans la production sociologique contemporaine.

\section{AUTEURS}

\section{MARC JOLY}

Université de Versailles Saint-Quentin-en-Yvelines, Laboratoire Printemps - CNRS 